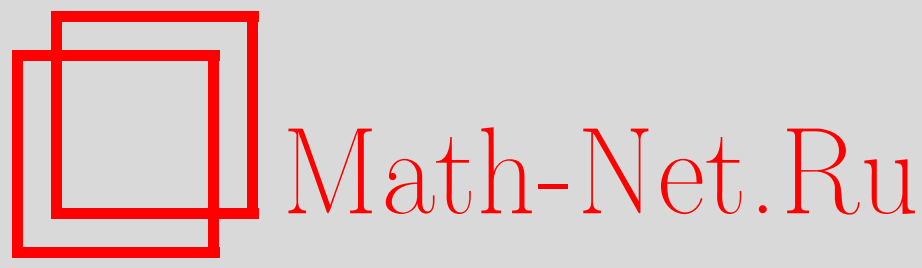

Е. П. Попова, К. В. Степаньянц, Формула для однопетлевых расходимостей в двух измерениях и ее применение для полей высших спинов, ТМФ, 2016, том 187, номер 3, 505-518

DOI: https://doi.org/10.4213/tmf9067

Использование Общероссийского математического портала Math-Net.Ru подразумевает, что вы прочитали и согласны с пользовательским соглашением http: //www . mathnet.ru/rus/agreement

Параметры загрузки:

IP : 54.196 .121 .252

26 апреля 2023 г., $17: 36: 17$

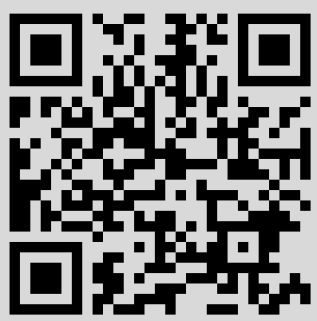




\title{
ФОРМУЛА ДЛЯ ОДНОПЕТЛЕВЫХ РАСХОДИМОСТЕЙ В ДВУХ ИЗМЕРЕНИЯХ И ЕЕ ПРИМЕНЕНИЕ ДЛЯ ПОЛЕЙ ВЫСШИХ СПИНОВ
}

\begin{abstract}
Получена простая формула для однопетлевых логарифмических расходимостей на фоне двумерного искривленного пространства-времени для теорий, в которых вторая вариация действия является неминимальным дифференциальным оператором второго порядка с малой неминимальностью. В частности, эта формула позволяет вычислять слагаемые, которые представляют собой интегралы от полных производных. В качестве ее применения найдены однопетлевые расходимости для полей высших спинов на фоне пространства постоянной кривизны в неминимальной калибровке, зависящей от двух параметров. Посредством явного вычисления показано, что с рассматриваемой точностью результат является калибровочно-независимым и, кроме того, не зависит от величины спина $s$ при $s \geqslant 3$.
\end{abstract}

Ключевые слова: однопетлевые расходимости, поля высших спинов.

DOI: $10.4213 / \operatorname{tmf} 9067$

\section{1. ВВЕДЕНИЕ}

Поиск однопетлевых расходимостей является одной из типичных вычислительных задач в квантовой теории поля. Стандартным способом ее решения является построение и расчет всех расходящихся диаграмм. Однако в некоторых случаях могут возникать определенные сложности. Например, при проведении вычислений в искривленном пространстве количество расходящихся диаграмм оказывается бесконечным, если теория возмущений строится на плоском фоне. Тем не менее запись результата в ковариантном виде позволяет определить сумму всех расходящихся вкладов и в этой ситуации. Ковариантный результат можно построить, к примеру, на основе расчета в пределе слабого поля, когда отклонение метрики от плоской считается малым, или какими-либо другими способами.

Работа К. В. Степаньянца поддержана РФФИ (грант № 14-01-00695).

* Московский государственный университет им. М. В. Ломоносова, Научно-исследовательский институт ядерной физики им. Д. В. Скобельцына, Москва, Россия

${ }^{\dagger}$ Московский государственный университет им. М. В. Ломоносова, физический факультет, Москва, Россия. E-mail: stepan@m9com.ru 
Однако представляется возможным существенно упростить вычисление однопетлевых расходимостей, если некоторые операции выполнить в общем виде, - например, как в методе, предложенном 'т Хофтом и Вельтманом [1], с помощью которого были впервые получены однопетлевые расходимости в гравитации. Суть данного метода заключается в том, что если вторая вариация действия (которая определяет однопетлевые расходимости) является минимальным дифференциальным оператором второго порядка, то можно построить ковариантную формулу, связывающую однопетлевые расходимости с коэффициентами в этом операторе. При этом не нужно в каждом конкретном случае восстанавливать ковариантный результат по разложению в приближении слабого поля. Таким образом, метод 'т Хофта-Вельтмана позволяет существенно упростить вычисление однопетлевых расходимостей и легко определять их знаки и коэффициенты. Основным его недостатком является наличие достаточно сильных ограничений на вид второй вариации действия. Посредством использования различных технических приемов был построен ряд обобщений этого метода. В частности, формула 'т Хофта-Вельтмана была обобщена на случай произвольного дифференциального оператора [2]. Несмотря на то что результат оказался очень громоздким, он был использован для однопетлевых вычислений в целом ряде случаев [3]-[7]. Кроме того, полученная общая формула дает возможность упростить автоматизацию вычислений при применении программ, позволяющих работать с тензорами (см., например, работы [8], [9]).

С математической точки зрения метод 'т Хофта-Вельтмана сводится к определению коэффициента $b_{4}$ Минакшисундарама-Сили-де Витта [10]-[13] для дифференциального оператора, совпадающего со второй вариацией действия. В четырех измерениях этот коэффициент связан с однопетлевыми логарифмическими расходимостями. В общем случае в пространстве размерности $d=2 n$ однопетлевые логарифмические расходимости связаны с коэффициентом $b_{2 n}$.

Для различных дифференциальных операторов коэффициенты Минакшисундарама-Сили-де Витта можно вычислить с помощью техники Швингера-де Витта и ее обобщений [14]-[17]. Однако такая техника не позволяет получить ответ в случае произвольного дифференциального оператора. С другой стороны, в работе [2] не были определены слагаемые, которые являются полными производными (т. е. дающие вклады в однопетлевые расходимости, которые являются интегралами от полных производных). Поэтому возникает вопрос, как обобщить результаты статьи [2], для того чтобы учесть слагаемые, представляющие собой полные производные. Такие вклады являются существенными в ряде случаев, например, если вычисления производятся на фоне пространств (анти-) де Ситтера ((А)дС). Отметим, что в этой ситуации, как правило, используются другие методы. Так, к примеру, для минимального оператора коэффициенты Минакшисундарама-Сили-де Витта можно найти в произвольной размерности, используя гармонический анализ на однородных пространствах [18]. Посредством этого метода однопетлевые расходимости для полей произвольного спина на фоне пространства $(\mathrm{A})$ дС в четырех измерениях были вычислены в статье [19] при использовании минимальной калибровки. Аналогичное вычисление, основанное на формуле для коэффициента $b_{4}$ минимального оператора, было выполнено в работе [20].

В настоящей статье мы пытаемся понять, как можно вычислять слагаемые, являющиеся полными производными, с использованием техники 'т Хофта-Вельтмана для неминимальных операторов. Для этого рассматривается наиболее простой слу- 
чай пространства двух измерений, когда логарифмические расходимости связаны с коэффициентом $b_{2}$. Мы определяем этот коэффициент, учитывая слагаемые, являющиеся полными производными, для неминимального оператора второго порядка, считая, что неминимальные слагаемые являются малыми, но отличными от 0. Затем этот результат проверяется посредством расчета однопетлевых расходимостей для теории высших спинов в неминимальной калибровке, зависящей от двух произвольных (малых) параметров.

Статья построена следующим образом. В разделе 2 рассматривается метод вычисления однопетлевых расходимостей, который применим в случае, когда вторая вариация действия является неминимальным дифференциальным оператором второго порядка с малыми неминимальными слагаемыми, и приводится результат этого вычисления, который дается формулой (4). В разделе 3 с помощью этого результата рассчитывается расходящаяся часть однопетлевого эффективного действия для теории высших спинов на фоне пространства (А)дС в случае двух измерений при использовании неминимальной двухпараметрической калибровки. Для этого в п. 3.1 напоминаются основные сведения теории высших спинов на фоне пространств постоянной кривизны. Расчет однопетлевых расходимостей проводится в п. 3.2. Ответ дается формулой (16). Полученные результаты кратко обсуждаются в разделе 4. Один их технических вопросов, связанный с проведением однопетлевого вычисления, рассматривается в приложении настоящей статьи.

\section{2. ОДНОПЕТЛЕВЫЕ РАСХОДИМОСТИ В ДВУХ ИЗМЕРЕНИЯХ ДЛЯ НЕМИНИМАЛЬНОГО ОПЕРАТОРА ВТОРОГО ПОРЯДКА}

В однопетлевом приближении эффективное действие записывается в виде (см., например, книгу [21])

$$
\Gamma[\varphi]=S[\varphi]+\frac{i}{2} \hbar \operatorname{Tr} \ln D+O\left(\hbar^{2}\right)
$$

где операция $\operatorname{Tr}$ по определению включает операцию $\int d^{d} x$, а дифференциальный оператор $D$ есть вторая вариация классического действия:

$$
D_{i}{ }^{j}=\frac{\delta^{2} S}{\delta \varphi^{i} \delta \varphi_{j}},
$$

где каждый из индексов $i$ и $j$ обозначает всю совокупность возможных индексов полей $\varphi$.

Большинство моделей в теории поля квадратичны по производным полей. Поэтому для многих приложений достаточно рассматривать только дифференциальные операторы второго порядка. Отметим, что в ряде случаев интерес представляют и дифференциальные операторы высших порядков (см., например, статью [22]). Произвольный неминимальный дифференциальный оператор второго порядка имеет вид

$$
D_{i}^{j}=\left(I g^{\mu \nu} \nabla_{\mu} \nabla_{\nu}+K^{\mu \nu} \nabla_{\mu} \nabla_{\nu}+S^{\mu} \nabla_{\mu}+W\right)_{i}{ }^{j},
$$

где для дальнейшего удобства мы выделили слагаемые, содержащие оператор Лапласа, а ковариантная производная записывается с помощью выражения

$$
\left(\nabla_{\mu}\right)_{i}{ }^{j}=\delta_{i}^{j} \partial_{\mu}+\omega_{\mu i}{ }^{j}
$$


в котором $\omega_{\mu i}{ }^{j}$ - некоторая связность. Коэффициенты $I_{i}{ }^{j}, K^{\mu \nu}{ }_{i}{ }^{j}, S^{\mu}{ }_{i}{ }^{j}$ и $W_{i}{ }^{j}$ представляют собой функции полей и получаются после вычисления второй вариации действия. При этом без ограничения общности можно считать, что величина $K^{\mu \nu}{ }_{i}^{j}$ симметрична по индексам $\mu$ и $\nu$ (как мы и предполагаем всюду далее). Термин "неминимальный" означает, что слагаемые с максимальным числом производных отличаются от оператора Лапласа, возведенного в некоторую степень (в данном случае речь идет о слагаемых со вторыми производными, которые нужно сравнивать с первой степенью оператора Лапласа).

Мы вычисляем однопетлевые расходимости, применяя размерную регуляризацию [23]-[26]. При этом размерность пространства-времени обозначается через $d$. В случае, если рассматривается теория в двух измерениях, то расходящаяся часть однопетлевого эффективного действия пропорциональна $(d-2)^{-1}$, причем считается, что $d \rightarrow 2$ после перенормировки. Используя инвариантность относительно общекоординатных преобразований, удобно представить расходящуюся часть однопетлевого эффективного действия в виде

$$
\Gamma_{1 \text { loop }}^{(\infty)}=\frac{1}{4 \pi(d-2)} \int d^{2} x \sqrt{-g} b_{2}
$$

где $b_{2}$ - некоторая ковариантная функция полей, являющаяся вторым коэффициентом Минакшисундарама-Сили-де Витта. В настоящей работе мы вычисляем эту величину для неминимального дифференциального оператора второго порядка, считая, что неминимальные слагаемые являются малыми. Кроме того, мы считаем, что верно равенство $\left(S^{\mu}\right)_{i}{ }^{j}=0$, справедливое в очень многих конкретных задачах.

Для подсчета однопетлевых расходимостей мы применяем обобщение метода, предложенного 'т Хофтом и Вельтманом [1], с помощью которого коэффициент $b_{4}$ был вычислен для произвольного дифференциального оператора без учета слагаемых, являющихся полными производными [2]. В настоящей работе мы учитываем слагаемые, которые представляют собой полные производные. Рассмотрим оператор

$$
D_{i}^{j}=\left(I g^{\mu \nu} \nabla_{\mu} \nabla_{\nu}+\varepsilon K^{\mu \nu} \nabla_{\mu} \nabla_{\nu}+W\right)_{i}{ }^{j},
$$

предполагая, что малый параметр $\varepsilon \rightarrow 0$. При этом результат для однопетлевых расходимостей вычисляется с точностью до величин первого порядка по $\varepsilon$. Без ограничения общности можно считать (что мы и делаем), что матрица $\varepsilon K_{i}^{\mu \nu}{ }_{i}$ является симметричной по индексам $\mu$ и $\nu$. Кроме того, мы также предполагаем, что эта матрица и матрица $I_{i}^{j}$ зависят только от метрического тензора $g_{\alpha \beta}$ (конечно, зависимость от $\delta_{\alpha}^{\beta}$ и $g^{\alpha \beta}$ тоже допустима).

Для расчета однопетлевых расходимостей мы используем диаграммную технику. Для того чтобы в рамках этой техники учесть слагаемые, являющиеся интегралами от полных производных, умножим логарифм оператора $D$ на некоторую не имеющую индексов вспомогательную функцию $a(x)$ и определим след результата:

$$
(\operatorname{Tr} a(x) \ln D)^{(\infty)}=\left(\int d^{2} x a(x) \operatorname{tr} \ln D\right)^{(\infty)}=\frac{2}{i} \frac{1}{4 \pi(d-2)} \int d^{2} x \sqrt{-g} a(x) b_{2},
$$

где $\operatorname{tr}$ обозначает обычный матричный след по индексам $i$ и $j$. Если положить $a(x)=1$, то с точностью до множителя $i / 2$ получится величина однопетлевого вклада в эффективное действие. Однако если требуется аккуратно учесть слагаемые, 
являющиеся интегралами от полной производной, то необходимо наличие нетривиальной функции $a(x)$. Величину (2) можно представить в виде суммы однопетлевых диаграмм, на одной из внешних линий в которых расположена функция $a(x)$. Для этого удобно записать оператор $D$ следующим образом:

$$
D_{i}{ }^{j} \equiv I_{0 i}{ }^{j} \partial_{\mu}^{2}+\varepsilon K_{0}^{\mu \nu}{ }_{i}{ }^{j} \partial_{\mu} \partial_{\nu}+V_{i}{ }^{j},
$$

где матрицы $I_{0}$ и $K_{0}^{\mu \nu}$ получаются из матриц $I$ и $K^{\mu \nu}$ заменой метрического тензоpa $g_{\mu \nu}$ (или $\left.g^{\mu \nu}\right)$ на метрику плоского пространства $\eta_{\mu \nu}$ (или $\eta^{\mu \nu}$ ). Оператор $V$ при этом включает в себя все остальные слагаемые. Принимая во внимание равенство

$$
\ln \left(I_{0} \partial_{\mu}^{2}\right)=\ln I_{0}+\ln \partial_{\mu}^{2}
$$

построим диаграммы, соответствующие выражению (2), используя разложение

$$
\begin{aligned}
\ln D= & \ln \left(I_{0} \partial_{\mu}^{2}\right)+\ln \left(1+\frac{1}{\partial_{\mu}^{2}} I_{0}^{-1}\left(\varepsilon K_{0}^{\mu \nu} \partial_{\mu} \partial_{\nu}+V\right)\right)+ \\
+ & \frac{1}{2}\left[\ln I_{0}+\ln \partial_{\mu}^{2}, \ln \left(1+\frac{1}{\partial_{\mu}^{2}} I_{0}^{-1}\left(\varepsilon K_{0}^{\mu \nu} \partial_{\mu} \partial_{\nu}+V\right)\right)\right]+ \\
+ & \frac{1}{12}\left[\ln I_{0}+\ln \partial_{\mu}^{2},\left[\ln I_{0}+\ln \partial_{\mu}^{2}, \ln \left(1+\frac{1}{\partial_{\mu}^{2}} I_{0}^{-1}\left(\varepsilon K_{0}^{\mu \nu} \partial_{\mu} \partial_{\nu}+V\right)\right)\right]\right]+ \\
+ & \frac{1}{12}\left[\ln \left(1+\frac{1}{\partial_{\mu}^{2}} I_{0}^{-1}\left(\varepsilon K_{0}^{\mu \nu} \partial_{\mu} \partial_{\nu}+V\right)\right),\right. \\
& \left.\quad\left[\ln \left(1+\frac{1}{\partial_{\mu}^{2}} I_{0}^{-1}\left(\varepsilon K_{0}^{\mu \nu} \partial_{\mu} \partial_{\nu}+V\right)\right), \ln I_{0}+\ln \partial_{\mu}^{2}\right]\right]+\cdots,
\end{aligned}
$$

в которых троеточие обозначает слагаемые с бо́льшим числом коммутаторов.

Первое слагаемое в выражении (3) не зависит от полей. Его вклад в однопетлевые расходимости представляет собой некоторую несущественную постоянную. Остальные слагаемые содержат член

$$
\ln \left(1+\frac{1}{\partial_{\mu}^{2}} I_{0}^{-1}\left(\varepsilon K_{0}^{\mu \nu} \partial_{\mu} \partial_{\nu}+V\right)\right)
$$

который можно легко разложить в ряд по $V$ и $\varepsilon$. Все слагаемые, пропорциональные нулевой и первой степени $V$ или $\varepsilon$, содержащие коммутатор с $\ln I_{0}$, исчезают после взятия матричного следа, поскольку функция $a(x)$ не имеет матричных индексов.

Оставшиеся слагаемые дают сумму диаграмм Фейнмана в однопетлевом приближении. Как уже отмечалось, на одной из внешних линий в этих диаграммах расположена функция $a(x)$. Из этих диаграмм выбираются расходящиеся. Если вычисления проводятся на фоне плоского пространства-времени, то имеется только одна расходящаяся диаграмма, приведенная на рис. 1а.

С помощью формул (2) и (3) для этих диаграмм строятся аналитические выражения, из которых затем выделяются логарифмически расходящиеся слагаемые. Как обычно, они вычисляются в евклидовом пространстве после поворота Вика. В рассматриваемых слагаемых выполняется интегрирование по углам, после чего 


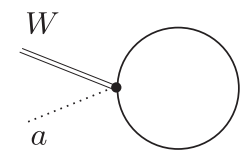

a

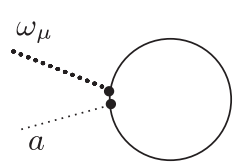

б

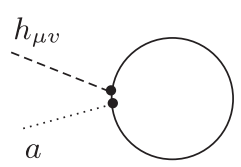

B

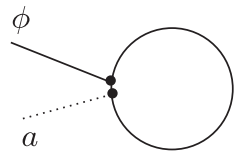

$\Gamma$

Рис. 1. Диаграммы в размерности $d=2$, определяющие расходящуюся часть однопетлевого эффективного действия.

оставшийся евклидов интеграл по импульсу заменяется в соответствии с предписанием

$$
\int \frac{d^{d} k}{(2 \pi)^{2} k^{2}} \longrightarrow-\frac{1}{2 \pi(d-2)}
$$

Для того чтобы обобщить полученный результат на случай искривленного пространства-времени, удобно использовать разложение по слабому полю вблизи метрики плоского пространства-времени. Для этого мы определяем величину $h_{\mu \nu} \equiv g_{\mu \nu}-\eta_{\mu \nu}$, которая является отклонением метрики от плоской метрики $\eta^{\mu \nu}$. Ее мы считаем малой. Число однопетлевых расходящихся диаграмм, в которых поле $h_{\mu \nu}$ расположено на внешних линиях, бесконечно. Однако сумма этих диаграмм представляет собой разложение в ряд по слабому полю некоторого ковариантного результата. Исходя из размерных соображений, несложно убедиться, что слагаемые, которые могут появиться при вычислении однопетлевых расходимостей, должны содержать кривизну в не более чем первой степени. Поэтому из всего бесконечного набора расходящихся диаграмм достаточно вычислить только диаграммы на рис. 1б-1г. При этом два маленьких смежных кружка означают, что между, например, $h_{\mu \nu}$ и $a$ могут стоять производные $\partial_{\mu}$. Через $\phi^{a}$ (как и в работе [2]) обозначены поля $h_{\alpha \beta}$, которые возникают при разложении $\varepsilon K^{\mu \nu}{ }_{i}{ }^{j}$ по отклонению метрики от плоской. Эти поля исключаются из окончательного результата с помощью тождества

$$
0=\nabla_{\alpha} K_{i}^{\mu \nu}{ }_{i}^{j}=\frac{\partial}{\partial \phi^{a}} K_{i}^{\mu \nu}{ }_{i}^{j} \partial_{\alpha} \phi^{a}+\Gamma_{\alpha \beta}^{\mu} K^{\beta \nu}{ }_{i}^{j}+\Gamma_{\alpha \beta}^{\nu} K_{i}^{\mu \beta}{ }_{i}^{j}+\omega_{\alpha i}{ }^{k} K^{\mu \nu}{ }_{k}^{j}-K^{\mu \nu}{ }_{i}{ } \omega_{\alpha k}{ }^{j} .
$$

Вычисляя диаграммы на рис. 1а-1г посредством описанного выше алгоритма, а затем восстанавливая ковариантный результат с помощью формул

$$
\begin{gathered}
R_{\mu \nu}=\frac{1}{2}\left(\partial_{\mu} \partial_{\alpha} h_{\nu}{ }^{\alpha}+\partial_{\nu} \partial_{\alpha} h_{\mu}{ }^{\alpha}-\partial_{\mu} \partial_{\nu} h_{\alpha}{ }^{\alpha}-\partial^{2} h_{\mu \nu}\right)+O\left(h^{2}\right), \\
R=\partial_{\mu} \partial_{\nu} h^{\mu \nu}-\partial^{2} h_{\alpha}{ }^{\alpha}+O\left(h^{2}\right), \\
F_{\mu \nu i}{ }^{j}=\partial_{\mu} \omega_{\nu i}{ }^{j}-\partial_{\nu} \omega_{\mu i}{ }^{j}+O\left(\omega^{2}\right),
\end{gathered}
$$

окончательно получаем, что

$$
b_{2}=\operatorname{tr}\left(\widehat{W}+\frac{1}{6} R-\frac{1}{2} \varepsilon \widehat{K}_{\alpha}{ }^{\alpha} \widehat{W}-\frac{1}{12} \varepsilon \widehat{K}_{\alpha}{ }^{\alpha} R+\frac{1}{6} \varepsilon \widehat{K}^{\mu \nu} R_{\mu \nu}\right),
$$

где используются обозначения $\varepsilon \widehat{K}^{\mu \nu} \equiv I^{-1} \varepsilon K^{\mu \nu}, \widehat{W} \equiv I^{-1} W$. Тот факт, что результат удается записать в ковариантном виде, можно рассматривать как нетривиальную проверку правильности проведенных вычислений. Также заметим, что полученный результат имеет очень компактный вид (по сравнению с выражением для 
однопетлевых расходимостей в случае произвольного неминимального оператора), благодаря чему можно предположить, что в пределе $\varepsilon \rightarrow 0$ формулу для однопетлевых расходимостей в четырехмерном пространстве можно существенно упростить.

При $\varepsilon K^{\mu \nu}{ }_{i}{ }^{j}=\varepsilon g^{\mu \nu} \delta_{i}^{j}$ и $I=1$ формула (4) воспроизводит известный результат для коэффициента $b_{2}$ минимального оператора (см., например, статью [27]) с учетом слагаемых, являющихся полными производными.

\section{3. ОДНОПЕТЛЕВЫЕ РАСХОДИМОСТИ ДЛЯ ПОЛЕЙ ВЫСШИХ СПИНОВ В НЕМИНИМАЛЬНОЙ КАЛИБРОВКЕ}

3.1. Поля высших спинов на фоне пространства (А)дС. Высшие спины описываются полностью симметричными тензорными полями $\phi_{\mu_{1} \mu_{2} \ldots \mu_{s}}$, которые удовлетворяют условию двойной бесследовости $\phi_{\alpha}{ }^{\alpha}{ }_{\beta}{ }^{\beta} \mu_{5} \ldots \mu_{s}=0$ (в настоящей работе мы рассматриваем случай $s \geqslant 3$ ). На фоне плоского пространства-времени свободное действие для таких полей было построено в работе [28] (фермионные поля высших спинов и действие для них приведены в статье [29]). Построение теории высших спинов с взаимодействием возможно [30], [31], но оказывается очень сложной задачей. Даже записать квадратичное по полям высших спинов действие на фоне искривленного пространства-времени [32] удается только для случая, когда фоновая геометрия представляет собой пространство постоянной кривизны (пространство (А)дС), для которого

$$
R_{\mu \nu \alpha \beta}=\frac{1}{d(d-1)}\left(g_{\mu \alpha} g_{\nu \beta}-g_{\mu \beta} g_{\nu \alpha}\right) R
$$

где $R=$ const. В статье [33] было показано, что при определенных предположениях непротиворечивая лагранжева формулировка для свободных бозонных полностью симметричных полей высших спинов возможна только в этой ситуации. На фоне пространства (А)дС действие для полей высших спинов записывается в виде

$$
\begin{aligned}
S= & \frac{(-1)^{s}}{2} \int d^{d} x \sqrt{-g}\left[\left(\nabla_{\alpha} \phi_{\mu_{1} \ldots \mu_{s}}\right)^{2}-\frac{1}{2} s(s-1)\left(\nabla_{\alpha} \phi_{\beta}{ }^{\beta} \mu_{3} \ldots \mu_{s}\right)^{2}-\right. \\
& -s\left(\nabla^{\alpha} \phi_{\alpha \mu_{2} \ldots \mu_{s}}\right)^{2}+s(s-1) \nabla_{\alpha} \phi^{\alpha \beta \mu_{3} \ldots \mu_{s}} \nabla_{\beta} \phi_{\gamma}{ }^{\gamma}{ }_{\mu_{3} \ldots \mu_{s}}- \\
& \left.-\frac{1}{4} s(s-1)(s-2)\left(\nabla_{\alpha} \phi^{\alpha \beta}{ }_{\beta \mu_{4} \ldots \mu_{s}}\right)^{2}+c_{1} R\left(\phi_{\mu_{1} \ldots \mu_{s}}\right)^{2}+c_{2} R\left(\phi_{\gamma}{ }^{\gamma} \mu_{3} \ldots \mu_{s}\right)^{2}\right],
\end{aligned}
$$

где квадраты тензорных величин обозначают свертки с метрикой $g^{\mu \nu}$ по всем свободным индексам, а коэффициенты $c_{1}$ и $c_{2}$ равны соответственно

$$
c_{1}=-\frac{(s-1)(s-4)}{d(d-1)}-\frac{(s-2)}{d}, \quad c_{2}=\frac{s(s-1)}{2 d}\left(s-1+\frac{s(s-3)}{d-1}\right) .
$$

Эти значения получаются из требования инвариантности действия относительно преобразований $\delta \phi_{\mu_{1} \ldots \mu_{s}}=\left(\nabla_{\mu_{1}} \alpha_{\mu_{2} \ldots \mu_{s}}+\nabla_{\mu_{2}} \alpha_{\mu_{1} \mu_{3} \ldots \mu_{s}}+\cdots\right) / s$, параметром которых является полностью симметричный тензор $\alpha_{\mu_{1} \mu_{2} \ldots \mu_{s-1}}$, удовлетворяющий условию бесследовости $\alpha_{\beta}{ }^{\beta}{ }_{\mu_{3} \ldots \mu_{s-1}}=0$. В частном случае $d=2$, который изучается в на- 
стоящей работе, значения $c_{1}$ и $c_{2}$ оказываются равными

$$
c_{1}=-\frac{1}{2}\left(s^{2}-4 s+2\right), \quad c_{2}=\frac{s(s-1)}{4}\left(s^{2}-2 s-1\right) .
$$

Для квантования калибровочных теорий [34] (и, в частности, рассматриваемой теории высших спинов на фоне пространства (А)дС) необходимо зафиксировать калибровку и добавить соответствующие духи Фаддеева-Попова [35]. Хорошо известно, что эффективное действие не зависит от выбора калибровки при использовании уравнения движения. Однако явные вычисления (см., например, статью [36]) показывают, что вне массовой поверхности эффективное действие зависит от выбора калибровочного условия. В настоящей работе калибровка фиксируется добавлением слагаемых

$$
\begin{aligned}
S_{\mathrm{gf}}= & \frac{(-1)^{s}}{2} \int d^{d} x \sqrt{-g} s(1+\lambda)\left(\nabla^{\alpha} \phi_{\alpha \mu_{1} \ldots \mu_{s-1}}-\frac{1}{2}(s-1)(1+\beta) \nabla_{\left(\mu_{1}\right.} \phi_{\left.\alpha \mu_{2} \ldots \mu_{s-1}\right)}^{\alpha}+\right. \\
& \left.+\frac{\beta(s-1)(s-2)}{2 d+4(s-3)} g_{\left(\mu_{1} \mu_{2}\right.} \nabla_{\alpha} \phi_{\left.\beta \mu_{3} \ldots \mu_{s-1}\right)}^{\alpha \beta}\right)^{2}
\end{aligned}
$$

причем последнее слагаемое добавлено для того, чтобы условие калибровки было бесследовым и число калибровочных условий совпало с числом параметров калибровочных преобразований. Круглые скобки обозначают симметризацию по индексам $\mu_{1} \mu_{2} \ldots \mu_{s-1}$. В общем случае

$$
T_{\left(\mu_{1} \mu_{2} \ldots \mu_{k}\right)} \equiv \frac{1}{k !}\left(T_{\mu_{1} \mu_{2} \ldots \mu_{k}}+T_{\mu_{2} \mu_{1} \ldots \mu_{k}}+\right.\text { другие перестановки индексов). }
$$

Совершая калибровочное преобразование в выражении

$$
\begin{aligned}
\nabla^{\alpha} \phi_{\alpha \mu_{1} \ldots \mu_{s-1}} & -\frac{1}{2}(s-1)(1+\beta) \nabla_{\left(\mu_{1}\right.} \phi^{\alpha}{ }_{\left.\alpha \mu_{2} \ldots \mu_{s-1}\right)}+ \\
+ & \frac{\beta(s-1)(s-2)}{2 d+4(s-3)} g_{\left(\mu_{1} \mu_{2}\right.} \nabla_{\alpha} \phi_{\left.\beta \mu_{3} \ldots \mu_{s-1}\right)}^{\alpha \beta}
\end{aligned}
$$

для калибровочного условия, стандартным образом получаем лагранжиан для духов Фаддеева-Попова. При этом калибровочный параметр становится духовым полем $c_{\mu_{1} \mu_{2} \ldots \mu_{s-1}}$, а результат умножается на антидуховое поле $\bar{c}^{\mu_{1} \mu_{2} \ldots \mu_{s-1}}$. Как и параметры калибровочного преобразования, духовые и антидуховые поля являются полностью симметричными и бесследовыми. Несложно убедиться, что результат для духового лагранжиана записывается в виде

$$
\begin{aligned}
L_{\mathrm{gh}}= & \bar{c}^{\mu_{1} \mu_{2} \ldots \mu_{s-1}}\left(\nabla^{\alpha} \nabla_{\alpha} c_{\mu_{1} \mu_{2} \ldots \mu_{s-1}}-\frac{\beta}{2}(s-1)\left(\nabla_{\mu_{1}} \nabla^{\alpha}+\nabla^{\alpha} \nabla_{\mu_{1}}\right) c_{\alpha \mu_{2} \ldots \mu_{s-1}}+\right. \\
& \left.+(\beta+2) \frac{(s-1)(d+s-3)}{2 d(d-1)} R c_{\mu_{1} \mu_{2} \ldots \mu_{s-1}}\right) .
\end{aligned}
$$

3.2. Вычисление однопетлевых расходимостей. В настоящей работе мы используем формулу (4) для вычисления расходящейся части однопетлевого эффективного действия в рассматриваемой теории в случае, когда $d=2, s \geqslant 3$, а параметры $\lambda$ и $\beta$ являются малыми. Тогда в низшем порядке по $\lambda$ и $\beta$ сумму классического 
действия и членов, фиксирующих калибровку, можно записать в виде

$$
\begin{aligned}
S & +S_{\mathrm{gf}}=\frac{(-1)^{s}}{2} \int d^{2} x \sqrt{-g}\left\{\left(\nabla_{\alpha} \phi_{\mu_{1} \mu_{2} \ldots \mu_{s}}\right)^{2}-\right. \\
& -\frac{1}{4} s(s-1)(1-\lambda-2 \beta)\left(\nabla_{\alpha} \phi^{\beta}{ }_{\beta \mu_{1} \ldots \mu_{s-2}}\right)^{2}+\frac{\lambda s}{2}\left(\left(\nabla^{\alpha} \phi_{\alpha \mu_{1} \ldots \mu_{s-1}}\right)^{2}+\right. \\
& \left.+\nabla_{\alpha} \phi_{\beta \mu_{1} \ldots \mu_{s-1}} \nabla^{\beta} \phi^{\alpha \mu_{1} \ldots \mu_{s-1}}\right)-s(s-1)(\lambda+\beta) \nabla^{\alpha} \phi_{\alpha \beta \mu_{1} \ldots \mu_{s-2}} \nabla^{\beta} \phi_{\gamma} \gamma \mu_{1} \ldots \mu_{s-2}+ \\
& +\frac{1}{8} s(s-1)(s-2)(\lambda+2 \beta)\left(\left(\nabla_{\alpha} \phi_{\beta \mu_{1} \ldots \mu_{s-3}}^{\alpha \beta}\right)^{2}+\right. \\
& \left.+\nabla_{\gamma} \phi_{\beta \mu_{1} \ldots \mu_{s-3}}^{\alpha \beta} \nabla_{\alpha} \phi_{\delta} \delta \gamma \mu_{1} \ldots \mu_{s-3}\right)+R\left(\phi_{\mu_{1} \mu_{2} \ldots \mu_{s}}\right)^{2}\left(-\frac{s^{2}-4 s+2}{2}+\frac{\lambda s^{2}}{4}\right)+ \\
& \left.+\frac{1}{4} R\left(\phi_{\alpha \mu_{1} \ldots \mu_{s-2}}^{\alpha}\right)^{2} s(s-1)\left(s^{2}-2 s-1-\lambda-\frac{1}{4}(s-2)^{2}(2+\lambda+2 \beta)\right)+o(\lambda, \beta)\right\},
\end{aligned}
$$

где $o(\lambda, \beta)$ обозначает слагаемые более высокого порядка по $\lambda$ и $\beta$.

Вторая вариация этого выражения по полям $\phi_{\alpha_{1} \alpha_{2} \ldots \alpha_{s}}$ является дифференциальным оператором второго порядка. Вычисляя след логарифма этого оператора, мы получаем однопетлевые диаграммы, в которых по петле распространяется поле спина $s$, а на внешних линиях стоит поле $h_{\mu \nu}$. Однако вычислять диаграммы в данном случае не нужно, поскольку можно воспользоваться формулой (4), которая сразу дает сумму их расходящихся частей в ковариантном виде. Для того чтобы воспользоваться этой формулой, необходимо найти вторую вариацию (7), а затем построить по ней выражения для матриц $I, \varepsilon K^{\mu \nu}$ и $W$. При этом важно учесть, что поля $\phi_{\alpha_{1} \alpha_{2} \ldots \alpha_{s}}$ удовлетворяют условию двойной бесследовости. В результате во всех матрицах появляются проекторы на дважды бесследовые состояния. Мы будем обозначать их через $Q_{\alpha_{1} \alpha_{2} \ldots \alpha_{s}} \beta_{1} \beta_{2} \ldots \beta_{s}$. Структура и свойства таких проекторов обсуждаются в приложении настоящей статьи.

После вычисления второй вариации действия получаем, что она представляет собой дифференциальный оператор вида (1), причем (после отбрасывания несущественной мультипликативной постоянной) справедливы следующие равенства:

$$
\begin{aligned}
I_{\alpha_{1} \alpha_{2} \ldots \alpha_{s}}{ }^{\beta_{1} \beta_{2} \ldots \beta_{s}}= & Q_{\alpha_{1} \alpha_{2} \ldots \alpha_{s}}{ }^{\gamma_{1} \gamma_{2} \ldots \gamma_{s}}\left(1_{\gamma_{1} \gamma_{2} \ldots \gamma_{s}}^{\delta_{1} \delta_{2} \ldots \delta_{s}}-\frac{s(s-1)}{4} g_{\left(\gamma_{1} \gamma_{2}\right.} g^{\left(\delta_{1} \delta_{2}\right.} 1_{\left.\gamma_{3} \ldots \gamma_{s}\right)}^{\left.\delta_{3} \ldots \delta_{s}\right)}\right) \times \\
& \times Q_{\delta_{1} \delta_{2} \ldots \delta_{s}}^{\beta_{1} \beta_{2} \ldots \beta_{s}}, \\
\varepsilon K_{\alpha_{1} \alpha_{2} \ldots \alpha_{s}}^{\beta_{1} \beta_{2} \ldots \beta_{s}}= & Q_{\alpha_{1} \alpha_{2} \ldots \alpha_{s}}{ }^{\gamma_{1} \gamma_{2} \ldots \gamma_{s}}\left\{\frac{s(s-1)}{4}(\lambda+2 \beta) g^{\mu \nu} g_{\left(\gamma_{1} \gamma_{2}\right.} g^{\left(\delta_{1} \delta_{2}\right.} 1_{\left.\gamma_{3} \ldots \gamma_{s}\right)}^{\left.\delta_{3} \ldots \delta_{s}\right)}+\right. \\
& +\frac{s \lambda}{2}\left(\delta_{\left(\gamma_{1}\right.}^{\mu} g^{\nu\left(\delta_{1}\right.} 1_{\left.\gamma_{2} \ldots \gamma_{s}\right)}^{\left.\delta_{2} \ldots \delta_{s}\right)}+\delta_{\left(\gamma_{1}\right.}^{\nu} g^{\mu\left(\delta_{1}\right.} 1_{\left.\gamma_{2} \ldots \gamma_{s}\right)}^{\left.\delta_{2} \ldots \delta_{s}\right)}\right)- \\
& -\frac{s(s-1)}{2}(\lambda+\beta)\left(\delta_{\left(\gamma_{1}\right.}^{\mu} \delta_{\gamma_{2}}^{\nu} g^{\left(\delta_{1} \delta_{2}\right.} 1_{\left.\gamma_{3} \ldots \gamma_{s}\right)}^{\left.\delta_{3} \ldots \delta_{s}\right)}+\right. \\
& \left.+g^{\mu\left(\delta_{1}\right.} g^{\nu \delta_{2}} g_{\left(\gamma_{1} \gamma_{2}\right.} 1_{\left.\gamma_{3} \ldots \gamma_{s}\right)}^{\left.\delta_{3} \ldots \delta_{s}\right)}\right)+ \\
& +\frac{s(s-1)(s-2)}{8}(\lambda+2 \beta)\left(\delta_{\left(\gamma_{1}\right.}^{\mu} g^{\nu\left(\delta_{1}\right.} g^{\delta_{2} \delta_{3}} g_{\gamma_{2} \gamma_{3}} 1_{\left.\gamma_{4} \ldots \gamma_{s}\right)}^{\left.\delta_{4} \ldots \delta_{s}\right)}+\right. \\
& \left.\left.+\delta_{\left(\gamma_{1}\right.}^{\nu} g^{\mu\left(\delta_{1}\right.} g^{\delta_{2} \delta_{3}} g_{\gamma_{2} \gamma_{3} 1_{\left.\gamma_{4} \ldots \gamma_{s}\right)}}^{\left.\delta_{4} \ldots \delta_{s}\right)}\right)\right\} Q_{\delta_{1} \delta_{2} \ldots \delta_{s}}^{\beta_{1} \beta_{2} \ldots \beta_{s}}
\end{aligned}
$$




$$
\begin{aligned}
W_{\alpha_{1} \alpha_{2} \ldots \alpha_{s}}^{\beta_{1} \beta_{2} \ldots \beta_{s}}= & Q_{\alpha_{1} \alpha_{2} \ldots \alpha_{s}}{ }^{\gamma_{1} \gamma_{2} \ldots \gamma_{s}}\left\{R\left(\frac{1}{2}\left(s^{2}-4 s+2\right)-\frac{1}{4} \lambda s^{2}\right) 1_{\gamma_{1} \gamma_{2} \ldots \gamma_{s}}^{\delta_{1} \delta_{2} \ldots \delta_{s}}-\right. \\
& -\frac{1}{4} R s(s-1)\left(s^{2}-2 s-1-\lambda-\frac{1}{4}(s-2)^{2}(2+\lambda+2 \beta)\right) \times \\
& \left.\times g_{\left(\gamma_{1} \gamma_{2}\right.} g^{\left(\delta_{1} \delta_{2}\right.} 1_{\left.\gamma_{3} \ldots \gamma_{s}\right)}^{\left.\delta_{3} \ldots \delta_{s}\right)}\right\} Q_{\delta_{1} \delta_{2} \ldots \delta_{s}}^{\beta_{1} \beta_{2} \ldots \beta_{s}}
\end{aligned}
$$

где используется обозначение $1_{\gamma_{1} \gamma_{2} \ldots \gamma_{k}}^{\beta_{1} \beta_{2} \ldots \beta_{k}} \equiv \delta_{\left(\gamma_{1}\right.}^{\left(\beta_{1}\right.} \delta_{\gamma_{2}}^{\beta_{2}} \ldots \delta_{\left.\gamma_{k}\right)}^{\left.\beta_{k}\right)}$. Матрица, обратная к $I$, определяется условием

$$
I_{\alpha_{1} \alpha_{2} \ldots \alpha_{s}}^{\beta_{1} \beta_{2} \ldots \beta_{s}}\left(I^{-1}\right)_{\beta_{1} \beta_{2} \ldots \beta_{s}}{ }^{\gamma_{1} \gamma_{2} \ldots \gamma_{s}}=Q_{\alpha_{1} \alpha_{2} \ldots \alpha_{s}}{ }^{\gamma_{1} \gamma_{2} \ldots \gamma_{s}},
$$

из которого можно получить явное выражение для матрицы $I^{-1}$, имеющее вид

$$
\left(I^{-1}\right)_{\alpha_{1} \alpha_{2} \ldots \alpha_{s}}^{\beta_{1} \beta_{2} \ldots \beta_{s}}=Q_{\alpha_{1} \alpha_{2} \ldots \alpha_{s}}{ }^{\gamma_{1} \gamma_{2} \ldots \gamma_{s}}\left(1_{\gamma_{1} \gamma_{2} \ldots \gamma_{s}}^{\beta_{1} \beta_{2} \ldots \beta_{s}}-\frac{s(s-1)}{4(s-2)} g_{\left(\gamma_{1} \gamma_{2}\right.} g^{\left(\beta_{1} \beta_{2}\right.} 1_{\left.\gamma_{3} \ldots \gamma_{s}\right)}^{\left.\beta_{3} \ldots \beta_{s}\right)}\right) .
$$

После подстановки матриц (8)-(10) и (11) в формулу (4) получается следующий результат для коэффициента $b_{2}$ второй вариации действия $(7)$ :

$$
b_{2(\text { main })}=\left(2(s-1)^{2}-\frac{4}{3}+\beta s(s-1)^{2}+o(\lambda, \beta)\right) R .
$$

Интеграл от этого выражения, умноженного на $\sqrt{-g}$, по $d^{2} x$ пропорционален расходящейся части суммы однопетлевых диаграмм Фейнмана, в которых по петле распространяется поле спина $s$.

Кроме того, в расходящуюся часть однопетлевого эффективного действия дают вклад диаграммы, в которых по петле распространяются духи Фаддеева-Попова. Этот вклад также можно вычислить с помощью формулы (4), которую нужно применить ко второй вариации духового действия. При $d=2$ соответствующий лагранжиан принимает вид

$$
\begin{aligned}
L_{\mathrm{gh}}= & \bar{c}^{\mu_{1} \mu_{2} \ldots \mu_{s-1}}\left(\nabla^{\alpha} \nabla_{\alpha} c_{\mu_{1} \mu_{2} \ldots \mu_{s-1}}-\frac{\beta}{2}(s-1)\left(\nabla_{\mu_{1}} \nabla^{\alpha}+\nabla^{\alpha} \nabla_{\mu_{1}}\right) c_{\alpha \mu_{2} \ldots \mu_{s-1}}+\right. \\
& \left.+\frac{1}{4}(\beta+2)(s-1)^{2} R c_{\mu_{1} \mu_{2} \ldots \mu_{s-1}}\right) .
\end{aligned}
$$

Принимая во внимание полную симметрию и бесследовость духовых полей, несложно убедиться, что матрицы, необходимые для вычислений с помощью формулы (4), имеют следующий вид:

$$
\begin{gathered}
\varepsilon K_{\alpha_{1} \ldots \alpha_{s-1}}^{\mu \nu} \beta_{1} \ldots \beta_{s-1}=-\frac{1}{2} \beta(s-1) P_{\alpha_{1} \ldots \alpha_{s-1}} \gamma_{1} \ldots \gamma_{s-1} \times \\
\times\left[\left(g^{\mu\left(\delta_{1}\right.} \delta_{\left(\gamma_{1} 1_{\gamma_{2} \ldots \gamma_{s-1}}^{\nu} 1_{2} \ldots \delta_{s-1}\right)}^{\delta_{2}}+g^{\nu\left(\delta_{1}\right.} \delta_{\left(\gamma_{1}\right.}^{\mu} 1_{\left.\gamma_{2} \ldots \gamma_{s-1}\right)}^{\left.\delta_{2} \ldots \delta_{s-1}\right)}\right] P_{\delta_{1} \ldots \delta_{s-1}} \beta_{1} \ldots \beta_{s-1},\right. \\
W_{\alpha_{1} \ldots \alpha_{s-1}}{ }^{\beta_{1} \ldots \beta_{s-1}}=\frac{1}{4}(\beta+1)(s-1)^{2} R P_{\alpha_{1} \ldots \alpha_{s-1}} \beta_{1} \ldots \beta_{s-1},
\end{gathered}
$$

где $P_{\alpha_{1} \ldots \alpha_{s-1}} \beta_{1} \ldots \beta_{s-1}$ - проектор на бесследовые состояния в двух измерениях. Его структура, а также некоторые его свойства описаны в приложении к настоящей статье. 
Подставляя матрицы (14) и (15) в формулу (4), после несложных преобразований получаем, что для диаграмм, в которых по петле распространяются духи Фаддеева-Попова, выражение для коэффициента $b_{2}$ имеет вид

$$
b_{2(\mathrm{gh})}=\left((s-1)^{2}+\frac{1}{3}+\frac{s(s-1)^{2}}{2} \beta+o(\beta)\right) R .
$$

Объединим результаты для основного вклада и вклада духов Фаддеева-Попова. При этом необходимо учесть, что духовые поля антикоммутируют, что дает дополнительный множитель - 1 , и отличаются от антидухов, что дает дополнительный множитель 2. Поэтому окончательный результат для расходящейся части однопетлевого эффективного действия при $s \geqslant 3$ записывается как

$$
\begin{aligned}
\Gamma_{1 \text {-loop }}^{(\infty)} & =\frac{1}{4 \pi(d-2)} \int d^{2} x \sqrt{-g}\left(b_{2(\text { main })}-2 b_{2(\text { gh })}\right)= \\
& =\frac{1}{4 \pi(d-2)} \int d^{2} x \sqrt{-g}(-2 R+o(\lambda, \beta)) .
\end{aligned}
$$

Это выражение не содержит слагаемых первого порядка по $\lambda$ и $\beta$. Поэтому в рассматриваемом приближении результат является калибровочно-независимым. Кроме того, результат также не зависит от значения $s$. При $s=3$ все полученные формулы переходят в соответствующие формулы работы [37], в которой был рассмотрен этот частный случай.

\section{4. ЗАКЛЮЧЕНИЕ}

В данной работе предлагается алгоритм для вычисления однопетлевых расходимостей в пространстве двух измерений в случае, когда вторая вариация действия представляет собой неминимальный оператор второго порядка, а "неминимальные" слагаемые являются малыми. При этом важно, что предлагаемая формула позволяет вычислять слагаемые, которые являются полными производными. Эта формула в рассматриваемом пределе оказалась очень простой. Она явно ковариантна и позволяет легко проводить вычисления на фоне искривленного пространства.

В качестве приложения мы вычислили однопетлевые расходимости для теории высших спинов на фоне пространства постоянной кривизны в неминимальной калибровке, зависящей от двух параметров $\lambda$ и $\beta$, в пределе, когда эти параметры являются малыми. При этом явным вычислением было продемонстрировано, что в рассматриваемом приближении результат является калибровочно-независимым. Это следует из того, что используемое эффективное действие представляет собой производящий функционал функций Грина при выключенных источниках, который от калибровки не зависит. Кроме того, проведенные вычисления показали независимость результата от величины спина $s$ при $s \geqslant 3$. Сокращение калибровочной зависимости также можно рассматривать как проверку правильности формулы (4), особенно если учесть, что все промежуточные выражения зависят от параметров калибровки крайне нетривиальным образом.

Несмотря на то что случай $d=2$ не столь интересен, как случай $d=4$, метод, использованный в настоящей работе, можно применить и для проведения вычислений в других размерностях. При этом в рассматриваемом пределе (когда неминимальные слагаемые малы) разумно ожидать, что ответ окажется намного проще, чем 
общая формула, приведенная в работе [2]. Кроме того, оказывается возможным учесть полные производные, которые в работе [2] были проигнорированы. Можно также попытаться получить ответ для произвольного неминимального оператора, для которого неминимальные слагаемые уже не считаются малыми, принимая во внимание слагаемые, которые являются полными производными. Однако пока эта задача не решена.

\section{ПРИЛОЖЕНИЕ}

\section{Проекторы на бесследовые и дважды бесследовые состояния и их свойства}

Поскольку поля высших спинов являются дважды бесследовыми, а соответствующие духовые поля - бесследовыми, в выражениях для второй вариации действия (или действия для духов) появляются проекторы на дважды бесследовые (или бесследовые) состояния. В данном приложении мы описываем структуру этих проекторов и указываем некоторые их свойства.

Проектор на бесследовые состояния в двух измерениях в явном виде записывается KaK

$$
\begin{aligned}
P_{\alpha_{1} \ldots \alpha_{s-1}}^{\beta_{1} \ldots \beta_{s-1}}= & 1_{\alpha_{1} \ldots \alpha_{s-1}}^{\beta_{1} \ldots \beta_{s-1}}-x_{1} g_{\left(\alpha_{1} \alpha_{2}\right.} g^{\left(\beta_{1} \beta_{2}\right.} 1_{\left.\alpha_{3} \ldots \alpha_{s-1}\right)}^{\left.\beta_{3} \ldots \beta_{s-1}\right)}- \\
& -x_{2} g_{\left(\alpha_{1} \alpha_{2}\right.} g_{\alpha_{3} \alpha_{4}} g^{\left(\beta_{1} \beta_{2}\right.} g^{\beta_{3} \beta_{4}} 1_{\left.\alpha_{5} \ldots \alpha_{s-1}\right)}^{\left.\beta_{5} \ldots \beta_{s-1}\right)}-\cdots,
\end{aligned}
$$

где $x_{1}, x_{2}, \ldots$ - некоторые числовые коэффициенты, зависящие от $s$ и $d$. Явные выражения для этих коэффициентов в настоящей работе не требуются. Их можно найти, например, в работе [20]. Бесследовый проектор обладает следующими свойствами:

$$
\begin{gathered}
P_{\alpha_{1} \alpha_{2} \ldots \alpha_{s-1}}^{\beta_{1} \beta_{2} \ldots \beta_{s-1}} g^{\alpha_{1} \alpha_{2}}=0, \quad P_{\alpha_{1} \alpha_{2} \ldots \alpha_{s-1}}^{\beta_{1} \beta_{2} \ldots \beta_{s-1}} g_{\beta_{1} \beta_{2}}=0, \\
P_{\alpha_{1} \alpha_{2} \ldots \alpha_{s-1}}{ }^{\beta_{1} \beta_{2} \ldots \beta_{s-1}} P_{\beta_{1} \beta_{2} \ldots \beta_{s-1}}{ }^{\gamma_{1} \gamma_{2} \ldots \gamma_{s-1}}=P_{\alpha_{1} \alpha_{2} \ldots \alpha_{s-1}} \gamma_{1} \gamma_{2} \ldots \gamma_{s-1}
\end{gathered}
$$

При этом первые два равенства фактически представляют собой часть определения проектора, а последнее немедленно следует из них. Кроме того, если $d=2$ при $s \geqslant 2$, то

$$
\operatorname{tr} P=P_{\alpha_{1} \ldots \alpha_{s-1}}{ }^{\alpha_{1} \ldots \alpha_{s-1}}=2,
$$

в чем можно легко убедиться, вычисляя число независимых компонент бесследового поля в двух измерениях.

Аналогичным образом проектор на дважды бесследовые состояния имеет вид

$$
\begin{aligned}
Q_{\alpha_{1} \alpha_{2} \ldots \alpha_{s}}^{\beta_{1} \beta_{2} \ldots \beta_{s}}= & 1_{\alpha_{1} \alpha_{2} \ldots \alpha_{s}}^{\beta_{1} \beta_{2} \ldots \beta_{s}}-y_{1} g_{\left(\alpha_{1} \alpha_{2}\right.} g_{\alpha_{3} \alpha_{4}} g^{\left(\beta_{1} \beta_{2}\right.} g^{\beta_{3} \beta_{4}} 1_{\left.\alpha_{5} \ldots \alpha_{s}\right)}^{\left.\beta_{5} \ldots \beta_{s}\right)}- \\
& -y_{2} g_{\left(\alpha_{1} \alpha_{2}\right.} g_{\alpha_{3} \alpha_{4}} g_{\alpha_{5} \alpha_{6}} g^{\left(\beta_{1} \beta_{2}\right.} g^{\beta_{3} \beta_{4}} g^{\beta_{5} \beta_{6}} 1_{\left.\alpha_{7} \ldots \alpha_{s}\right)}^{\left.\beta_{7} \ldots \beta_{s}\right)}-\cdots
\end{aligned}
$$

где $y_{1}, y_{2}, \ldots$ - некоторые числовые коэффициенты, зависящие от $s$ и $d$, и удовлетворяет следующим свойствам:

$$
\begin{gathered}
Q_{\alpha_{1} \alpha_{2} \ldots \alpha_{s}}^{\beta_{1} \beta_{2} \ldots \beta_{s}} g^{\alpha_{1} \alpha_{2}} g^{\alpha_{3} \alpha_{4}}=0, \quad Q_{\alpha_{1} \alpha_{2} \ldots \alpha_{s}}^{\beta_{1} \beta_{2} \ldots \beta_{s}} g_{\beta_{1} \beta_{2}} g_{\beta_{3} \beta_{4}}=0 \\
Q_{\alpha_{1} \alpha_{2} \ldots \alpha_{s}}^{\beta_{1} \beta_{2} \ldots \beta_{s}} Q_{\beta_{1} \beta_{2} \ldots \beta_{s}}^{\gamma_{1} \gamma_{2} \ldots \gamma_{s}}=Q_{\alpha_{1} \alpha_{2} \ldots \alpha_{s}}^{\gamma_{1} \gamma_{2} \ldots \gamma_{s}}
\end{gathered}
$$


В случае $d=2$ также справедливы следующие равенства:

$$
\begin{gathered}
\operatorname{tr} Q=Q_{\alpha_{1} \alpha_{2} \ldots \alpha_{s}}{ }^{\alpha_{1} \alpha_{2} \ldots \alpha_{s}}=4, \quad \text { если } s \geqslant 3, \\
Q_{\alpha_{1} \alpha_{2} \ldots \alpha_{s}}{ }^{\beta_{1} \beta_{2} \ldots \beta_{s}} g^{\alpha_{1} \alpha_{2}} g_{\beta_{1} \beta_{2}}=\frac{4}{s} P_{\alpha_{3} \ldots \alpha_{s}}{ }^{\beta_{3} \ldots \beta_{s}} .
\end{gathered}
$$

При этом след проектора $Q$ можно получить посредством вычисления количества независимых компонент дважды бесследового поля в двух измерениях. Для проверки последнего равенства мы замечаем, что левая часть, очевидно, пропорциональна бесследовому проектору, а коэффициент можно определить сравнением слагаемых, которые пропорциональны $1_{\alpha_{3} \ldots \alpha_{s}}^{\beta_{3} \ldots \beta_{s}}$.

Благодарности. Авторы выражают глубокую благодарность И. Л. Бухбиндеру, С. М. Кузенко, П. И. Пронину и А.А. Решетняку за полезные замечания и обсуждения.

\section{Список литературы}

[1] G. 't Hooft, M. Veltman, Ann. Inst. H. Poincaré Sect. A (N. S.), 20 (1974), 69-74.

[2] P. I. Pronin, K.V. Stepanyantz, Nucl. Phys. B, 485:1 (1997), 517-544, arXiv: hep-th/ 9605206.

[3] K. A. Kazakov, P. I. Pronin, K. V. Stepanyantz, Gravit. Cosmology, 4:1 (1998), 17-27.

[4] M. Y. Kalmykov, K. A. Kazakov, P. I. Pronin, K. V. Stepanyantz, Class. Quant. Grav., 15:12 (1998), 3777-3794, arXiv: hep-th/9809169.

[5] K. A. Kazakov, P.I. Pronin, Phys. Rev. D, 59:6 (1999), 064012, 13 pp., arXiv: hep-th/ 9806023.

[6] К. А. Казаков, П. И. Пронин, ТМФ, 121:3 (1999), 387-411.

[7] Е. А. Андрияш, К. В. Степаньянц, Вестник Моск. ун-та. Сер. 3. Физ. Астрон., 2003, № $2,24-26$.

[8] D. A. Bolotin, S. V. Poslavsky, Introduction to Redberry: the computer algebra system designed for tensor manipulation, arXiv: 1302.1219.

[9] S. Poslavsky, D. Bolotin, J. Phys.: Conf. Ser., 608:1 (2015), 012060, 6 pp.

[10] S. Minakshisundaram, A. Pleijel, Canadian J. Math., 1 (1949), 242-256.

[11] S. Minakshisundaram, J. Indian Math. Soc. (N. S.), 17 (1953), 158-165.

[12] R. T. Seeley, "Complex powers of an elliptic operator", Singular Integrals (University of Chicago, Chicago, IL, April 20-22, 1966), Proceedings of Symposia in Pure Mathematics, 10, ed. A. P. Calderón, AMS, Providence, RI, 1967, 288-307.

[13] P. B. Gilkey, J. Diff. Geom., 10:4 (1975), 601-618.

[14] J. S. Schwinger, Phys. Rev., 82:5 (1951), 664-679.

[15] Б. С. де Витт, Динамическая теория групп и полей, М., Наука, 1987.

[16] A. O. Barvinsky, G. A. Vilkovisky, Phys. Rept., 119 (1985), 1.

[17] V.P. Gusynin, E. V. Gorbar, V. V. Romankov, Nucl. Phys. B, 362:1 (1991), 449-471.

[18] R. Camporesi, Phys. Rep., 196:1-2 (1990), 1-134.

[19] R. Camporesi, A. Higuchi, Phys. Rev. D, 47:8 (1993), 3339-3344.

[20] I. L. Buchbinder, V.A. Krykhtin, H. Takata, "One-loop divergence of effective action of Higher spin theory in $A d S_{4}$ ", talk given at the International Conference "Quantum Field Theory and Gravity" (Tomsk, July 5-9, 2010).

[21] К. Хуанг, Кварки, лептоны и калибровочные поля, Мир, М., 1985.

[22] P. I. Pronin, K. Stepanyantz, Phys. Lett. B, 414:1-2 (1997), 117-122, arXiv: hep-th/ 9707008. 
[23] G. 't Hooft, M. J. G. Veltman, Nucl. Phys. B, 44:1 (1972), 189-213.

[24] C. G. Bollini, J. J. Giambiagi, Nuovo Cimento B, 12:1 (1972), 20-26.

[25] J. F. Ashmore, Lett. Nuovo Cimento, 4:8 (1972), 289-290.

[26] G. M. Cicuta, E. Montaldi, Lett. Nuovo Cim., 4:9 (1972), 329-332.

[27] Y. N. Obukhov, Nucl. Phys. B, 212:2 (1983), 237-254.

[28] C. Fronsdal, Phys. Rev. D, 18:10 (1978), 3624-3629.

[29] J. Fang, C. Fronsdal, Phys. Rev. D, 18:10 (1978), 3630-3633.

[30] M. A. Vasiliev, Fortsch. Phys., 52:6 (2004), 702-717, arXiv: hep-th/0401177.

[31] X. Bekaert, S. Cnockaert, C. Iazeolla, M. A. Vasiliev, Nonlinear higher spin theories in various dimensions, arXiv: hep-th/0503128.

[32] C. Fronsdal, Phys. Rev. D, 20:4 (1979), 848-856.

[33] I. L. Buchbinder, V.A. Krykhtin, P. M. Lavrov, Modern Phys. Lett. A, 26:16 (2011), 1183-1196, arXiv: 1101.4860.

[34] А.А. Славнов, Л. Д. Фаддеев, Введение в квантовую теорию калибровочных полей, Наука, М., 1978.

[35] L. D. Faddeev, V. N. Popov, Phys. Lett. B, 25:1 (1967), 29-30.

[36] R. E. Kallosh, O. V. Tarasov, I. V. Tyutin, Nucl. Phys. B, 137:1-2 (1978), 145-163.

[37] Е. Попова, К. В. Степаньянц, Вестник ТГПУ, 13(128) (2012), 130-133.

Поступила в редакцию 16.10.2015 